\title{
Information Preservation in Statistical Privacy and Bayesian Estimation of Unattributed Histograms
}

\author{
Bing-Rong Lin \\ Dept. of Computer Science \& Engineering \\ Penn State University
}

\author{
Daniel Kifer \\ Dept. of Computer Science \& Engineering \\ Penn State University
}

\begin{abstract}
In statistical privacy, utility refers to two concepts: information preservation - how much statistical information is retained by a sanitizing algorithm, and usability - how (and with how much difficulty) does one extract this information to build statistical models, answer queries, etc. Some scenarios incentivize a separation between information preservation and usability, so that the data owner first chooses a sanitizing algorithm to maximize a measure of information preservation and, afterward, the data consumers process the sanitized output according to their needs $[22,46]$.

We analyze a variety of utility measures and show that the average (over possible outputs of the sanitizer) error of Bayesian decision makers forms the unique class of utility measures that satisfy three axioms related to information preservation. The axioms are agnostic to Bayesian concepts such as subjective probabilities and hence strengthen support for Bayesian views in privacy research. In particular, this result connects information preservation to aspects of usability - if the information preservation of a sanitizing algorithm should be measured as the average error of a Bayesian decision maker, shouldn't Bayesian decision theory be a good choice when it comes to using the sanitized outputs for various purposes? We put this idea to the test in the unattributed histogram problem where our decisiontheoretic post-processing algorithm empirically outperforms previously proposed approaches.
\end{abstract}

\section{Categories and Subject Descriptors}

H.2.8 [Database Applications]: Statistical databases

\section{Keywords}

privacy; utility; differential privacy; decision theory

\section{INTRODUCTION}

In order to protect privacy, a dataset containing sensitive information must be fed into a sanitizing algorithm that pro-

\footnotetext{
Permission to make digital or hard copies of all or part of this work for personal or classroom use is granted without fee provided that copies are not made or distributed for profit or commercial advantage and that copies bear this notice and the full citation on the first page. To copy otherwise, to republish, to post on servers or to redistribute to lists, requires prior specific permission and/or a fee.

Copyright 20XX ACM X-XXXXX-XX-X/XX/XX ...\$15.00.
}

duces sanitized data (e.g., perturbed/noisy datasets or query answers) which can be released to the public. The behavior of the sanitizing algorithm is guided by a privacy definition, such as differential privacy [16], to ensure that the sanitized data is indeed safe to release.

Many algorithms can satisfy a given privacy definition, but their utility will often differ. Some algorithms will preserve statistical information better than others, and some will produce outputs that are easier to use than others (as an extreme example, an algorithm that encrypts its input also perfectly preserves information, but this information is computationally difficult to access).

Some applications incentivize a separation between information preservation and usability. That is, they treat measures of information preservation as optimization criteria in algorithm design $[32,29,22,5,4,6]$. Once an algorithm is chosen, it is used to generate sanitized data and the data users must then process it to extract useful information.

One example is privacy-preserving data publishing, where the data owner plans a one-time publication of privacyenhanced data. In this case, the data owner seeks to maximize the information content of the sanitized data (subject to privacy constraints) and it is up to end-users, with the help of statistical experts, to process this data to build models, answer queries, etc.

Another example occurs when users have a fixed privacy budget [33] and must pay for privacy-preserving query answers $[30,21]$. In this case, the data user wants the data owner to run sanitizing algorithms that maximize information content subject to privacy and cost constraints.

Finally, there is theoretical [22] and empirical [46] evidence suggesting that maximizing information first and then postprocessing it in a variety of ways allows the sanitized data to be used effectively for multiple purposes.

In this paper, we study measures of information preservation for sanitizing algorithms and methods for processing the output of these algorithms. In particular, we present a new estimation procedure for the sorted histogram problem (also known as the unattributed histogram problem) introduced by Hay et al. [24]. This procedure is motivated by our results on how to define measures of information preservation. We now describe these contributions in more detail.

\subsection{Problematic Measures}

There have been many proposals for measuring utility (e.g., $[22,13,4,6,11])$. Which of these are suitable for measuring the amount of information preserved by a sanitizing algorithm? It is not always intuitive, and so this question 
must be answered using scientific criteria [27, 28]. Consider the following example.

EXAMPLE 1.1. A dataset contains responses to a true/false survey question. The data owner must choose between the following two sanitizing algorithms (both of which satisfy her privacy requirements):

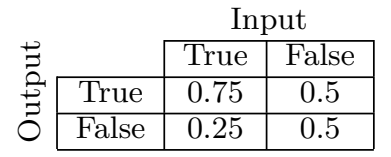

Algorithm $\mathfrak{M}_{1}$

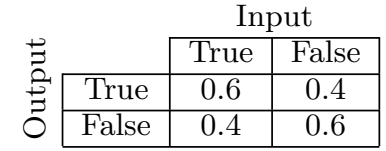

Algorithm $\mathfrak{M}_{2}$
Algorithm $\mathfrak{M}_{1}$ changes a "true" to "false" with probability 0.25 and changes "false" to "true" with probability 0.5 . Algorithm $\mathfrak{M}_{2}$ changes "true" to "false" and vice versa with probability 0.4. One popular utility measure $\mu$ is the negative of the worst-case error of a sanitizing algorithm. The maximum probability of error of $\mathfrak{M}_{1}$ is 0.5 (which occurs when the input is "false") and so its utility is $\mu\left(\mathfrak{M}_{1}\right)=-0.5$. The maximum probability of error of $\mathfrak{M}_{2}$ is 0.4 and so $\mu\left(\mathfrak{M}_{2}\right)=$ -0.4. Therefore $\mathfrak{M}_{2}$ has the higher utility score (-0.4 vs. -0.5) and would be chosen over $\mathfrak{M}_{1}$.

However, we claim that $\mathfrak{M}_{2}$ preserves less information than $\mathfrak{M}_{1}$. To see why, consider an algorithm $\mathcal{A}$ that changes a "true" to a "false" with probability 0.2 and never changes a "false" to "true". Then running $\mathfrak{M}_{2}$ on the data is equivalent to first running $\mathfrak{M}_{1}$ and then running $\mathcal{A}$ on the result (i.e. $\left.\mathfrak{M}_{2}=\mathcal{A} \circ \mathfrak{M}_{1}\right)$. Therefore if the output of $\mathfrak{M}_{2}$ can be used for an application then so can the output of $\mathfrak{M}_{1}$ (because we can always simulate $\mathfrak{M}_{2}$ by running $\mathcal{A}$ on the output of $\mathfrak{M}_{1}$ ). Thus in the target applications of this paper, $\mathfrak{M}_{1}$ should be preferred over $\mathfrak{M}_{2}$ and so worst case error is not suitable as a measure of information preservation.

A fundamental property [28] of a measure $\mu$ of information preservation is that $\mu\left(\mathfrak{M}_{2}\right) \leq \mu\left(\mathfrak{M}_{1}\right)$ whenever $\mathfrak{M}_{2}=\mathcal{A} \circ \mathfrak{M}_{1}$ for some randomized algorithm $\mathcal{A}$. That is, if $\mathfrak{M}_{2}$ can be simulated by first running $\mathfrak{M}_{1}$ on the sensitive data and then running $\mathcal{A}$ on the output of $\mathfrak{M}_{1}$, then $\mathfrak{M}_{1}$ provides more information than $\mathfrak{M}_{2}$.

Kifer and Lin [28] provided anecdotal evidence of utility measures that do not satisfy this property but did not provide a systematic analysis of existing utility measures. Thus, one of our contributions is an analysis of many existing utility measures to determine which ones are suitable measures of a sanitizing algorithm's ability to preserve information.

We note that in other contexts and applications, it can be necessary for a utility measure $\nu$ to violate this fundamental property - that is, it may be the case that $\mathfrak{M}_{2}=\mathcal{A} \circ \mathfrak{M}_{1}$ yet $\nu\left(\mathfrak{M}_{2}\right)>\nu\left(\mathfrak{M}_{1}\right)$. This can happen when $\mathfrak{M}_{1}$ or $\mathcal{A}$ are computationally expensive or difficult to implement (hence the data owner or user could prefer $\mathfrak{M}_{2}$ ); users might also prefer $\mathfrak{M}_{2}$ if its output is already in a format that they can directly use (or can be put into that format without too much effort), such as a model parameter. We refer to these concerns collectively as usability. More work needs to be done to quantify such tradeoffs between information preservation and usability (it is outside the scope of this paper).

\subsection{Axiomatically Justified Measures}

Can any information preservation measures for a sanitizing algorithm be justified from first principles? We analyzed three utility axioms presented by [28] and derived the following result: those axioms imply that the ability of a data sanitizer $\mathfrak{M}$ to preserve information should be measured as the average (over possible outputs of $\mathfrak{M}$ ) error of a Bayesian decision maker. This result is important because it provides support for a rarely used utility measure (we are only aware of its use by Alvim et al. [5, 4]).

Note that the axioms themselves are not Bayesian: they do not mention subjective probabilities nor Bayes' rule. They are also different from the types of axioms used in statistics and economics to justify Bayesian decision theory (e.g., [35, $44,43,34])$. Thus, although such a link should be expected to exist, it was not obvious that these particular axioms would provide the formal link between sanitizing algorithms and decision theory.

\subsection{Processing Sanitized Data}

End-users need to process sanitized data to extract query answers and build predictive models while taking advantage of probabilistic knowledge and constraints that are known to hold (e.g., [22, 23, 46, 7, 24, 31, 38, 49, 36, 3]). Since the ability of a sanitizing algorithm to preserve information should (according to the axioms) be measured as the expected error of a Bayesian decision maker, it stands to reason that this Bayesian methodology should play a more prominent role in the analysis of sanitized data

We apply this insight to the sorted histogram problem (also known as the unattributed histogram) first studied by Hay et al. [24]. The goal is to reconstruct a sorted histogram from differentially private sanitized data. The resulting sorted histogram can be used to study edge distributions in social networks, identify power laws, etc. [24]. Using hidden Markov models, we develop a reconstruction algorithm for the sorted histogram problem based on Bayesian decision theory. We show experimentally that our technique outperforms both the least-squares approach of Hay et al. [24] and maximum likelihood estimation.

\subsection{Summary of Contributions}

- In an attempt to identify measures of information preservation for sanitizing algorithms, we analyze many existing utility measures. Most of them have counterexamples similar to Example 1.1.

- We analyze the three utility axioms and show they imply that the ability of a data sanitizer $\mathfrak{M}$ to preserve information should be measured as the average (over possible outputs of $\mathfrak{M}$ ) error of a Bayesian decision maker. The axioms themselves are not Bayesian per se (e.g. they do not mention subjective probabilities).

- We develop a new estimation algorithm for the privacypreserving sorted histogram problem [24]. It is based on the idea that Bayesian decision theory should play a role in data processing since information preservation should be measured as the expected error of a Bayesian decision maker. A thorough experimental analysis shows that our algorithm consistently and significantly outperforms competing approaches. This serves as a partial empirical validation of the theoretical axiomatic approach.

\section{Open problems.}

We leave open the question of general methods for computing measures of information preservation and optimizing algorithms based on them (this is a long-standing open 
problem for almost all utility measures; currently almost all utility results in the literature depend on highly inefficient/intractable algorithms or customized hand-crafted analyses $[32,29,22,5,4,6])$. Another open problem is quantifying the tradeoff between usability and information preservation.

\subsection{Outline}

We discuss related work in Section 2, introduce notation and terminology in Section 3, and review utility axioms from [28] in Section 4. After this we present the main contributions of this paper. We systematically analyze many existing utility measures in Section 5 to check whether they measure information preservation. We briefly review Bayesian decision theory in Section 6, after which we show that the average error of a Bayesian decision maker is an axiomatically justifiable measure of a data sanitizer's ability to preserve information. We present our decision-theoretic algorithm for the sorted histogram problem in Section 7, experiments in Section 8, and conclusions in Section 9.

\section{RELATED WORK}

\subsection{Utility axioms for statistical privacy}

Kifer and Lin [27, 28] proposed three axioms for measures of information preservation for sanitizing algorithms to aid in the scientific analysis of utility in statistical privacy. The axioms are discussed in more detail in Section 4. While our work follows their research direction, we have significant contributions of our own - new analyses of these axioms (including a proof of their connection to Bayesian theory) and a new estimation algorithm for privacy-preserving sorted histograms. We believe these analyses will have impact in future work on algorithm design for statistical privacy.

\subsection{Bayesian decision theory}

Axiomatic justifications for Bayesian decision theory are well-studied in statistics and economics. There are differences between our results and those from statistics and economics (e.g., [35, 44, 43, 34]). The goals differ (i.e., how to assign a number to an algorithm vs. how a user should make decisions in the face of uncertainty) and the subjects of the axioms differ (i.e., an algorithm vs. a data user's preference over actions after seeing sanitized data). In a sense, our results extend prior work in that the axioms about algorithms imply the existence of actions (that an end-user must choose from after seeing sanitized data), user preferences over actions, priors, and behaviors (i.e., Bayesian updating) that are treated in other work (each paper infers the existence of some but not all of these components).

The mathematical existence of subjective prior probabilities was derived by de Finetti through the concepts of exchangeability and the Dutch Book argument [35]. Given objective probabilities and a choices for actions, von Neumann and Morgenstern [44] used axioms to show that a decision maker should take an action that maximizes expected utility. Savage [43] extended this result to show that a rational decision maker is mathematically equivalent to an individual who has subjective probabilities and chooses actions to maximize expected subjective utility. Myerson [34] proposed a set of axioms whose consequences are as follows: a user makes decisions by choosing a subjective prior, forms the posterior distribution upon seeing new evidence, and then takes an action to maximize expected utility (using the posterior to compute the expectation). The language of those axioms talks about prizes and lotteries. Prize $X$ corresponds to an action (i.e. using $X$ as a query answer or a model parameter) and lotteries refer to the possibility that a user may want to choose an action randomly instead of deterministically.

\subsection{Utility measures}

In the literature, the word utility is very closely associated with concepts we refer to as usability. That is, proposed techniques often follow the principle that a user should not have to treat sanitized data any differently from ordinary data (although, for power users, it is important to account for the additional uncertainty in sanitized data).

Early work on privacy considered utility to be a property of the sanitized data rather than a property of the data sanitizer. As a result, utility was often measured as some notion of "closeness" between the original data and sanitized data (see $[13,19,2]$ for references to numerous utility metrics of this type) or "closeness" of a statistical analysis conducted on both datasets (e.g., [40, 1], which do not use detailed knowledge of how the data sanitizer works). Choosing data sanitizers according to such metrics often leads to privacy vulnerabilities such as minimality attacks [47].

In other cases, utility was recognized as a property of the data sanitizer but often was only defined for a restricted class of data sanitizers. For example, if we only consider data sanitizers that coarsen tuple attributes, one can measure utility based on the number of coarsening operations (e.g., [26]) or if we only consider data sanitizers that add noise to query answers, one can measure variance of the noise (e.g., [2]) or probability that the noise exceeds a certain bound (see $[10,15,24,11,17]$ for variations).

One of the most fundamental results about utility for data sanitizers is due to Ghosh et al. [22]. They considered scenarios having the following four characteristics: (1) the data owner wants to answer a single counting query in a private manner; (2) each end-user has a prior distribution over possible true query answers; (3) each end-user has a personal similarity measure between possible true query answers and possible sanitized query answers; (4) each end-user wants the data owner to choose a data sanitizer that maximizes the end-user's expected similarity between true and sanitized query answers. Ghosh et al. showed that in many cases, the data owner should first achieve privacy using a data sanitizer called the Geometric mechanism [22] and then customize the result for each end-user by performing a lossy postprocessing that will maximize the end-user's personal utility measure (to improve usability). Later, Gupte and Sundararajan [23] extended this result to a class of minimax utility measures. Kifer and Lin [27] then investigated useful properties for measures of information preservation. They proposed several axioms and measures [27, 28] that avoid problems illustrated in Example 1.1. However, the measures proposed in $[27,28]$ were not suitable for practical applications since it was not clear how to choose one of the information preservation measures based on the application at hand.

\subsection{Using sanitized data}

Once sanitized data has been produced, a data analyst often wishes to estimate some quantity - a query answer, a 
model parameter, etc. The research community has recognized that to build a reliable estimator, it is often necessary to process sanitized data in special ways - to account for constraints that must hold (e.g., $[7,24,14]$ ), to incorporate knowledge of how the data sanitizer works (e.g., [46, 29, $18,39]$ ), and to account for specialized output formats that can be used to represent uncertainty (e.g., [49, 48, 25, 3]). Bayesian methods (and Bayesian decision theory in particular) are not often used. This paper provides a formal link between measures of information preservation and tools for working with sanitized data via this statistical methodology.

\section{NOTATION AND TERMINOLOGY}

Let $\mathbb{I}=\left\{D_{1}, D_{2}, \ldots\right\}$ be the input domain of possible datasets. Note that $\mathbb{I}$ is a countable set (since datasets must be encodable as finite bit strings on digital computers). A data sanitizer $\mathfrak{M}$ is a deterministic or randomized algorithm whose domain is $\mathbb{I}$ and whose range is countable (for the same reason $\mathbb{I}$ is countable). Let

$$
\vec{P}[\mathfrak{M}(\cdot)=\omega] \stackrel{\text { def }}{=}\left\langle P\left[\mathfrak{M}\left(D_{1}\right)=\omega\right], P\left[\mathfrak{M}\left(D_{2}\right)=\omega\right], \ldots\right\rangle
$$

denote the vector corresponding to the probabilities of generating $\omega \in \operatorname{range}(\mathfrak{M})$. Note that it is defined even for deterministic sanitizers. A privacy definition $\mathfrak{P r i v}$ is a set of data sanitizers, with input domain $\mathbb{I}$, all of which are trusted to protect the sensitive information contained in datasets from II. An information preservation measure is a function $\mu_{\mathbb{I}}$ that assigns a real number $\mu_{\mathbb{I}}(\mathfrak{M})$ to any data sanitizer $\mathfrak{M}$ with domain $\mathbb{I}$ (for simplicity we disallow utility values of $\pm \infty$ ).

The data owner has a sensitive dataset $D$ and can use any $\mathfrak{M} \in \mathfrak{P r i v}$ (i.e. any data sanitizer belonging to a prespecified $^{1}$ privacy definition $\left.\mathfrak{P r i v}\right)$ to create sanitized data that can be released to the public. In an effort to preserve statistical information, the data owner will try to choose the data sanitizer $\mathfrak{M} \in \mathfrak{P r i v}$ that maximizes $\mu_{\mathbb{I}}(\mathfrak{M})$. It is important that $\mu_{\mathbb{I}}$ properly captures the notion of "information".

Sometimes it is more convenient to talk about an information loss measure $\mathcal{L}_{\mathbb{I}}(\mathfrak{M})$, which is simply the negative of an information preservation measure (i.e. $\left.\mathcal{L}_{\mathbb{I}}(\mathfrak{M})=-\mu_{\mathbb{I}}(\mathfrak{M})\right)$.

\section{A REVIEW OF UTILITY AXIOMS}

In this section we review axioms of information preservation from $[27,28]$. Note that we do not present any new axioms - our contribution lies in new analyses of these axioms (Sections 5 and 6) and, especially, in a new data processing algorithm for the sorted histogram problem (Section 7 ).

Axiom 4.1. (Sufficiency [28]). An information preservation measure $\mu_{\mathbb{I}}$ (resp., loss measure $\mathcal{L}_{\mathbb{I}}$ ) should satisfy the relation $\mu_{\mathbb{I}}\left(\mathfrak{M}_{1}\right) \geq \mu_{\mathbb{I}}\left(\mathfrak{M}_{2}\right)$ (resp., $\mathcal{L}_{\mathbb{I}}\left(\mathfrak{M}_{1}\right) \leq \mathcal{L}_{\mathbb{I}}\left(\mathfrak{M}_{2}\right)$ ) whenever $\mathfrak{M}_{2}=\mathcal{A} \circ \mathfrak{M}_{1}$ for some (possibly randomized) algorithm $\mathcal{A}$ whose domain contains the range of $\mathfrak{M}_{1}$.

The axiom of sufficiency essentially states that postprocessing by an algorithm $\mathcal{A}$ cannot increase the amount of information that is preserved. It is a fundamental axiom for any information measure.

However, note that postprocessing may improve usability. For example, $\mathcal{A}$ may take some of the information contained in the output of $\mathfrak{M}_{1}$ and put it into a usable form, such as

\footnotetext{
${ }^{1}$ Note that our work is not specific to any privacy definition,
} so the choice of privacy definition is up to the data owner. a model parameter or query answer. As we show in Section 6 , Bayesian decision theory plays a key role in usability and leads to a new processing algorithm for the sorted histogram problem (Section 7).

Another useful axiom is continuity [28]. It states that small changes in the probabilistic ${ }^{2}$ behavior of $\mathfrak{M}$ result in small changes to the amount of information preserved. We rephrase it here using the following metric over the collection of data sanitizers with the same input domain.

Definition 4.2. (Metric over data sanitizers). Define the metric $\mathrm{d}_{\mathbb{I}}^{*}$ over the set of data sanitizers with the same input domain $\mathbb{I}=\left\{D_{1}, D_{2}, \ldots\right\}$ as follows:

$$
\mathrm{d}_{\mathbb{I}}^{*}\left(\mathfrak{M}_{1}, \mathfrak{M}_{2}\right)=\sup _{D \in \mathbb{I}} \sum_{\omega}\left|P\left[\mathfrak{M}_{1}(D)=\omega\right]-P\left[\mathfrak{M}_{2}(D)=\omega\right]\right|
$$

where the summation is over $\omega \in \operatorname{range}\left(\mathfrak{M}_{1}\right) \cup \operatorname{range}\left(\mathfrak{M}_{2}\right)$.

Axiom 4.3. (Continuity [28]). An information preservation measure $\mu_{\mathbb{I}}$ (resp., loss measure $\mathcal{L}_{\mathbb{I}}$ ) should be continuous with respect to the metric $\mathrm{d}_{\mathbb{I}}^{*}$.

The final axiom we consider is called branching [28]. It quantifies how much information we expect to lose if we are only told that $\mathfrak{M}$ has output either $\omega_{1}$ or $\omega_{2}$ (but we don't know which one). The branching axiom states that this decrease depends on the probabilities with which $\omega_{1}$ and $\omega_{2}$ could be generated but does not depend on events that did not occur (i.e., it does not depend on $\omega_{3}, \omega_{4}, \ldots$ ).

Axiom 4.4. (Branching [28]). An information preservation measure $\mu_{\mathbb{I}}$ should satisfy the relation

$$
\mu_{\mathbb{I}}(\mathfrak{M})=\mu_{\mathbb{I}}(\widetilde{\mathfrak{M}})+G\left(\vec{P}\left[\mathfrak{M}(\cdot)=\omega_{1}\right], \quad \vec{P}\left[\mathfrak{M}(\cdot)=\omega_{2}\right]\right)
$$

for some function $G$, where

- $\omega_{1}$ and $\omega_{2}$ are the first two elements in range(M) (any arbitrary ordering will suffice).

- $\widetilde{\mathfrak{M}}$ is an algorithm with range $(\widetilde{\mathfrak{M}})=\left\{\omega^{*}\right\} \cup \operatorname{range}(\mathfrak{M})$ and which behaves exactly like $\mathfrak{M}$ except that $\widetilde{\mathfrak{M}}$ outputs $\omega^{*}$ whenever $\mathfrak{M}$ would have output $\omega_{1}$ or $\omega_{2}$.

- $\vec{P}\left[\mathfrak{M}(\cdot)=\omega_{i}\right]$ denotes the vector $\left\langle P\left[\mathfrak{M}\left(D_{1}\right)=\omega_{i}\right]\right.$, $\left.P\left[\mathfrak{M}\left(D_{2}\right)=\omega_{i}\right], \ldots\right\rangle$ corresponding to probabilities of generating $\omega_{i}$.

A loss measure $\mathcal{L}_{\mathbb{I}}$ should satisfy the same type of relation.

\section{INFORMATION-PRESERVING PROPER- TIES OF EXISTING UTILITY MEASURES}

Which utility measures satisfy the sufficiency axiom (and therefore are optimization criteria for algorithm design when information retention is the main concern)? In this section we analyze several popular utility measures.

\subsection{Expected and worst-case discrepancy be- tween input and output}

We first consider two of the most popular classes of utility measures which compute the expected and worst-case discrepancy between the input and output of a data sanitizer $\mathfrak{M}$.

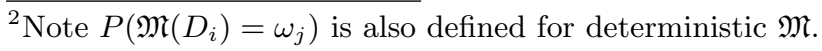


The components of these utility measures consist of a fixed set $\mathbb{O}^{*}$ of possible outputs, a probability distribution $P(D)$ over possible input datasets $D \in \mathbb{I}$ and either a quality function $Q$ or an error function $E$. When using quality functions, the quantity $Q(D, \omega)$ refers to the goodness of releasing sanitized output $\omega \in \mathbb{O}^{*}$ when the true input dataset is $D$ (the higher the better). When using error functions, the quantity $E(D, \omega)$ represents the dissimilarity between $D$ and $\omega$ (such as a squared distance between queries computed over $D$ and queries computed over $\omega$ ).

The expected discrepancy can be used as a loss measure by defining it as the expected value of $E$ (the expectation is with respect to the prior $P$ and randomness in the sanitizing algorithm) and as a utility measure by defining it as the expected value of $Q$ :

Definition 5.1. (Expected discrepancy). Given an input domain $\mathbb{I}$, fixed output domain $\mathbb{O}^{*}$, a quality function $Q: \mathbb{I} \times \mathbb{O}^{*} \rightarrow \mathbb{R}$ (for utility) or error function $E: \mathbb{I} \times \mathbb{O}^{*} \rightarrow$ $\mathbb{R}$ (for loss), and a prior distribution $P$ over $\mathbb{I}$, define the following utility $\mu_{\mathbb{I}}{ }^{S E}$ and loss $\mathcal{L}_{\mathbb{I}}{ }^{S E}$ measures (defined for all $\mathfrak{M}$ with range $\left.(\mathfrak{M}) \subseteq \mathbb{O}^{*}\right)$ as follows:

$$
\begin{aligned}
\mu_{\mathbb{I}}{ }^{S E}(\mathfrak{M}) & =\sum_{D \in \mathbb{I}} \sum_{\omega \in \mathbb{O}^{*}} Q(D, \omega) P(D) P(\mathfrak{M}(D)=\omega) \\
\mathcal{L}_{\mathbb{I}}{ }^{S E}(\mathfrak{M}) & =\sum_{D \in \mathbb{I}} \sum_{\omega \in \mathbb{O}^{*}} E(D, \omega) P(D) P(\mathfrak{M}(D)=\omega)
\end{aligned}
$$

On the other hand, instead of averaging over all inputs, one can simply drop the prior and consider the input that leads to the worst-case expected discrepancy (here the expectation is taken with respect to the randomness of a sanitizing algorithm, but not with respect to possible priors).

Definition 5.2. (Worst-case discrepancy). Given an input domain $\mathbb{I}$, fixed output domain $\mathbb{O}^{*}$, a quality function $Q: \mathbb{I} \times \mathbb{O}^{*} \rightarrow \mathbb{R}$ (for utility) or error function $E: \mathbb{I} \times \mathbb{O}^{*} \rightarrow$ $\mathbb{R}$ (for loss), define the utility $\mu_{\mathbb{I}}{ }^{S M}$ and loss $\mathcal{L}_{\mathbb{I}}{ }^{S M}$ measures (defined for all $\mathfrak{M}$ with range $(\mathfrak{M}) \subseteq \mathbb{O}^{*}$ ) as follows:

$$
\begin{aligned}
& \mu_{\mathbb{I}}{ }^{S M}(\mathfrak{M})=\min _{D \in \mathbb{I}} \sum_{\omega \in \mathbb{O}^{*}} Q(D, \omega) P(\mathfrak{M}(D)=\omega) \\
& \mathcal{L}_{\mathbb{I}}{ }^{S M}(\mathfrak{M})=\max _{D \in \mathbb{I}} \sum_{\omega \in \mathbb{O}^{*}} E(D, \omega) P(\mathfrak{M}(D)=\omega)
\end{aligned}
$$

For example, if each $\omega \in \mathbb{O}^{*}$ is interpreted as a query answer, set $E(D, \omega)$ to be 0 if the noisy answer $\omega$ has absolute error at most $\delta$ (i.e. the distance between the $\omega$ and the query answer computed from $D$ is at most $\delta$, for some pre-specified $\delta$ ); set $E(D, \omega)=1$ otherwise. The worst-case discrepancy then measures the worst-case (over all datasets) probability that $\mathfrak{M}$ did not answer the query accurately (i.e. with error at most $\delta$ ).

\subsubsection{Our analysis}

The following two theorems show that no nontrivial instantiations of expected and worst-case discrepancy measures can satisfy the sufficiency axiom.

THEOREM 5.3. A utility $\mu_{\mathbb{I}}{ }^{S E}$ or loss $\mathcal{L}_{\mathbb{I}}{ }^{S E}$ measure (as described in Definition 5.1) satisfies Axiom 4.1 (sufficiency) only if it is a constant function.

For proof see Appendix A.
TheOREM 5.4. Let II be finite. A utility $\mu_{\mathbb{I}}{ }^{S M}$ or loss $\mathcal{L}_{\mathbb{I}}{ }^{S M}$ measure (as described in Definition 5.2) satisfies Axiom 4.1 (sufficiency) only if it is a constant function.

For proof see Appendix B.

Thus they are not strictly measures of information preservation; they trade some information loss for other factors (such as usability). Quantifying this tradeoff is an interesting direction for future work.

\subsection{Expected error of most likely dataset}

The next utility measure we analyze can satisfy Axiom 4.1 in some nontrivial situations. It was used by Askari et al. [6], who observed that a sanitized output $\omega$ can provide information about the original inputs via maximum likelihood inference. Askari et al. [6] originally used this observation to define privacy loss, but the same formula can be used to define a utility measure as well.

The components of this measure are a prior probability distribution $P(D)$ over $D \in \mathbb{I}$ and an error measure $E(D, \widehat{D})$ that expresses the dissimilarity between two datasets $D$ and $\widehat{D}$. For example, $E(D, \widehat{D})$ could be 0 if $D=\widehat{D}$ and 1 otherwise. Another possibility is that $E(D, \widehat{D})$ could be the distance between a query answer computed from $D$ and a query answer computed from $\widehat{D}$. Suppose a data sanitizer $\mathfrak{M}$ produces an output $\omega$. The most likely input dataset is the one that maximizes $P(D) P(\mathfrak{M}(D)=\omega)$. Let us denote this most likely input as $\widehat{D}_{(\mathfrak{M}, \omega)}$. The loss measure of a data sanitizer $\mathfrak{M}$, as defined by $[6]$ is the expected error of $\widehat{D}_{(\mathfrak{M}, \omega)}$ :

DeFinition 5.5. (e.g., [6]). Given an input domain $\mathbb{I}$ and error function $E: \mathbb{I} \times \mathbb{I} \rightarrow \mathbb{R}$. The loss $\mathcal{L}_{\mathbb{I}}{ }^{M A P}(\mathfrak{M})$ is defined as

$$
\sum_{\omega \in \operatorname{range}(\mathfrak{M})} \sum_{D \in \mathbb{I}} P(D) P(\mathfrak{M}(D)=\omega) E\left(D, \widehat{D}_{(\mathfrak{M}, \omega)}\right)
$$

where $\widehat{D}_{(\mathfrak{M}, \omega)} \equiv \arg \max _{D \in \mathbb{I}} P(D) P(\mathfrak{M}(D)=\omega)$ is the maximum a posteriori dataset (i.e. has the highest posterior probability). For the corresponding utility measure $\mu_{\mathbb{I}}{ }^{M A P}$ replace the error function $E$ with a quality function $Q$.

\subsubsection{Our analysis}

For some choices of error function $E$ and quality function $Q$, the result is a nontrivial measure of information preservation.

TheOREM 5.6. The loss measure $\mathcal{L}_{\mathbb{I}}{ }^{M A P}(\mathfrak{M})$ described in Definition 5.5 satisfies Axiom 4.1 if and only if the corresponding error function $E$ has the form

$$
E(D, \widehat{D})=\alpha H(D, \widehat{D})+f(D)
$$

where $\alpha$ is a nonnegative constant, $f$ is an arbitrary function, and $H(D, \widehat{D})=0$ when $D=\widehat{D}$ and equals 1 otherwise. The condition for the utility measure $\mu_{\mathbb{I}}{ }^{M A P}$ is that the corresponding quality function $Q$ has the form

$$
Q(D, \widehat{D})=\alpha(1-H(D, \widehat{D}))+f(D)
$$

The " $+f(D)$ " term in Theorem 5.6 only plays a trivial role. Its effect is to add a constant (i.e. the expected value $\left.\sum_{D} f(D) P(D)\right)$ to the utility/loss of every algorithm $\mathfrak{M}$. Similarly, the $\alpha$ is just a constant factor. Thus, without loss of generality, one can restrict attention to error functions $E$ 
such that $E(D, \widehat{D})=1$ when $D \neq \widehat{D}$ and 0 otherwise. In these cases, $\mathcal{L}_{\mathbb{I}}{ }^{\mathrm{MAP}}(\mathfrak{M})$ is simply the probability of incorrectly guessing the true input.

\section{AXIOMATICALLY DERIVED MEASURES}

In this section, we present the second major contribution of this paper. We show that any loss measure $\mathcal{L}_{\mathbb{I}}$ that satisfies Axiom 4.1 (sufficiency), Axiom 4.3 (continuity), and Axiom 4.4 (branching) is precisely the expected error (over all possible outputs of a data sanitizer $\mathfrak{M}$ ) of a Bayesian decision maker.

This class of loss measures is rarely used in statistical privacy (we are only aware of work by Alvim et al. [5, 4]). Our contribution is the result that these loss measures are precisely the ones that satisfy those 3 axioms. Without presupposing subjective probabilities it, strengthens the case for wider use of Bayesian methods in statistical privacy. In particular, this result motivated our algorithm for the sorted histogram problem (Section 7) since it implies that Bayesian decision theory is an appropriate methodology for dealing with the output produced by sanitizing algorithms (i.e. optimizing algorithms for information preservation becomes equivalent to optimizing the error of a Bayesian decision maker who will process the data).

We first review Bayesian decision theory and then we present our result.

\subsection{Review of Bayesian decision theory, spe- cialized to sanitized data}

Bayesian decision theory is usually presented in very general language because it can be applied to many different types of problems. Since the context of interest is sanitized data, we specialize our discussion to how a user would use Bayesian decision theory to process sanitized data. The components of Bayesian decision theory are thus:

- A set $\mathbb{I}$ of possible datasets.

- A prior probability distribution over $\mathbb{I}$.

- A set $\mathbb{A}$ of actions. This corresponds to the type of statistical analysis a user wants to perform. For example, if a user wants an estimate of an answer to a query, then $\mathbb{A}$ could be the set of possible query answers. The user's job is to select the best action $a \in \mathbb{A}$ based on uncertain/probabilistic information (e.g. the user's job is to estimate the answer to a query based on a noisy output from a data sanitizer).

- An error function $\mathcal{E}$. The value $\mathcal{E}(D, a)$ corresponds to the error suffered by a user who selects action $a \in \mathbb{A}$ when the true (unknown) data is $D$. In the cases of query answers, $a$ would be a candidate answer to a query and $\mathcal{E}(D, a)$ could be the absolute difference between the query answer $a$ and the query answer computed from $D$.

- An output $\omega$ produced by a data sanitizer $\mathfrak{M}$ that was run on the true data (which is unknown to the user).

The subjective expected error of selecting an action $a \in \mathbb{A}$ after observing sanitized data $\omega$ is

$$
\begin{aligned}
& \sum_{D \in \mathbb{I}} \mathcal{E}(D, a) P(\text { data }=D \mid \mathfrak{M}(\text { data })=\omega) \\
& \quad=\sum_{D \in \mathbb{I}} \mathcal{E}(D, a) \frac{P(\mathfrak{M}(D)=\omega) P(D)}{\sum_{D^{\prime} \in \mathbb{I}} P\left(\mathfrak{M}\left(D^{\prime}\right)=\omega\right) P\left(D^{\prime}\right)}
\end{aligned}
$$

According to Bayesian decision theory, the user should choose an action that minimizes this expected error. Noting that the denominator is the same for all actions, this means that the optimal action $a^{*}$ is:

$$
a^{*}=\arg \min _{a \in \mathbb{A}} \sum_{D \in \mathbb{I}} \mathcal{E}(D, a) P(\mathfrak{M}(D)=\omega) P(D)
$$

Other Bayesian and frequentist methodologies are also possible $[20,45]$. However, our results in the next section indicate that choosing sanitizing algorithms to maximize information preservation is in some sense equivalent to choosing an algorithm whose outputs should be processed using Bayesian decision theory.

\subsection{Expected error of a Bayesian decision maker: an axiomatically justified loss measure}

When a user choose an action $a \in \mathbb{A}$ (such as a specific estimate for query answers or model parameters) using Equation 3, the expected error of this action-selection strategy depends on a sanitizing algorithm $\mathfrak{M}$ and thus can be viewed as a loss measure $\mathcal{L}_{\mathbb{I}}{ }^{\mathrm{BDT}}$ :

Definition 6.1. (Expected error of a Bayesian decision maker). Given an input domain $\mathbb{I}$, a prior over $\mathbb{I}$, a set $\mathbb{A}$ of actions, and an error function $\mathcal{E}: \mathbb{I} \times \mathbb{A} \rightarrow \mathbb{R}$ that is bounded from below (i.e. for all $D$ and all $a, \mathcal{E}(D, a) \geq \beta$ for some $\beta)$ define the loss measure $\mathcal{L}_{\mathbb{I}}{ }^{B D T}$ as:

$$
\mathcal{L}_{\mathbb{I}}{ }^{B D T}(\mathfrak{M})=\sum_{\omega \in \operatorname{range}(\mathfrak{M})} \inf _{a \in \mathbb{A}} \sum_{D \in \mathbb{I}} P(D) P(\mathfrak{M}(D)=\omega) \mathcal{E}(D, a)
$$

The error function has to be bounded below so that $\mathcal{L}_{\mathbb{I}}{ }^{\mathrm{BDT}}$ is a real number (and not $-\infty$ ). Also note that we can get a utility measure by taking the negative (i.e. $-\mathcal{L}_{\mathbb{I}}^{\mathrm{BDT}}(\mathfrak{M})$ ).

The next theorem says that $\mathcal{L}_{\mathbb{I}}{ }^{\mathrm{BDT}}$ avoids information paradoxes (such as Example 1.1) by satisfying Axiom 4.1 for all priors and all $\mathcal{E}$ that are bounded from below. More importantly, it shows that any loss measure that satisfies Axioms 4.1, 4.3, and 4.4 is equivalent to $\mathcal{L}_{\mathbb{I}}{ }^{\mathrm{BDT}}$ for some set of actions $\mathbb{A}$, some error function $\mathcal{E}$ (that is bounded from below), and some prior distribution $P(D)$.

TheOREM 6.2. Let II be finite.

(i) For every choice of $\mathbb{A}$, prior over $\mathbb{I}$, and error function $\mathcal{E}$ that is bounded from below, the resulting loss measure $\mathcal{L}_{\mathbb{I}}{ }^{B D T}$ (Definition 6.1) satisfies Axioms 4.1, 4.3, and 4.4.

(ii) If a loss measure $\mathcal{L}_{\mathbb{I}}$ satisfies Axioms 4.1, 4.3, and 4.4 then there exists a prior over $\mathbb{I}$, a set of actions $\mathbb{A}$, and an error function $\mathcal{E}$ (that is bounded from below) such the corresponding loss measure $\mathcal{L}_{\mathbb{I}}{ }^{B D T}$ equals $\mathcal{L}_{\mathbb{I}}$.

See Appendix C for the proof. 


\subsection{Discussion}

Traditionally, after seeing a sanitized output $\omega$, Bayesian decision theory defines the expected error as:

$$
\inf _{a \in \mathbb{A}} \sum_{D \in \mathbb{I}} \mathcal{E}(D, a) \frac{P(\mathfrak{M}(D)=\omega) P(D)}{P(\omega)}
$$

(where $P(\omega)=\sum_{D \in \mathbb{I}} P(D) P(\mathfrak{M}(D)=\omega)$ ). When we discuss expected error as a loss measure, we further must take the expectation with respect to $\omega$ as well (yielding the equation in Definition 6.1).

Note that measuring these quantities for sanitizing algorithms is generally a difficult problem as is optimizing algorithms based on these utility/loss measures. However, the same is true for almost all other types of utility and loss measures (the most common result is a hand-crafted asymptotic upper bound). Thus identifying tractable situations is an interesting area for future work.

We also expect that Bayesian methods for analyzing sanitized data will pose additional research challenges due to the type of noise that is added by the sanitization process [46]. We provide two pieces of evidence that research effort in this direction is worthwhile. The first is this theoretical argument that information preservation is the expected error of an analyst who uses Bayesian decision theory. The second is an empirical evaluation showing that our estimation algorithm for the sorted histogram problem outperforms prior non-Bayesian approaches. We describe the estimation algorithm in Section 7 and evaluate it in Section 8.

\section{ALGORITHM FOR THE SORTED HIS- TOGRAM PROBLEM}

We can put the theoretical results of Section 6 to the test by applying Bayesian decision theory to a problem in the analysis of sanitized data. The target application is the sorted histogram problem, introduced by Hay et al. [24].

\subsection{The Sorted Histogram Problem}

A sorted histogram, also known as the unattributed histogram [24], is a histogram in which the attribute names are not important so that the histogram buckets are sorted by their counts and the attribute labels are thrown away (for example, if the histogram counts are $\langle 5,1,0,3\rangle$, the corresponding sorted histogram $S$ is $\langle 0,1,3,5\rangle)$. It can be used to study structural properties of discrete distributions such as identifying power laws. For further motivation and a discussion of applications, see [24].

When privacy is a concern, the sorted histogram can be protected with $\epsilon$-differential privacy by adding independent Laplace $(1 / \epsilon)$ noise (with density $f(x)=\frac{\epsilon}{2} e^{-\epsilon|x|}$ ) to each cell [24] to get a noisy histogram $\tilde{S}$. The counts in this noisy version no longer appear in sorted order, so the challenge is to derive an estimator $\widehat{S}$ of the underlying sorted histogram $S$. Thus the counts in $\widehat{S}$ must also be in increasing order.

This is where usability plays an important role. The noisy histogram $\tilde{S}$ contains much information but it is not very useful for a user who wants to study the underlying sorted histogram. Thus to help such a user, we need to extract from $\tilde{S}$ a good estimate of the original histogram.

Hay et al. [24] proposed an estimator $\widehat{S}_{\mathrm{LS}}$ whose squared distance from $\tilde{S}$ is smallest (using order-constrained leastsquares regression). Accuracy can be improved slightly by

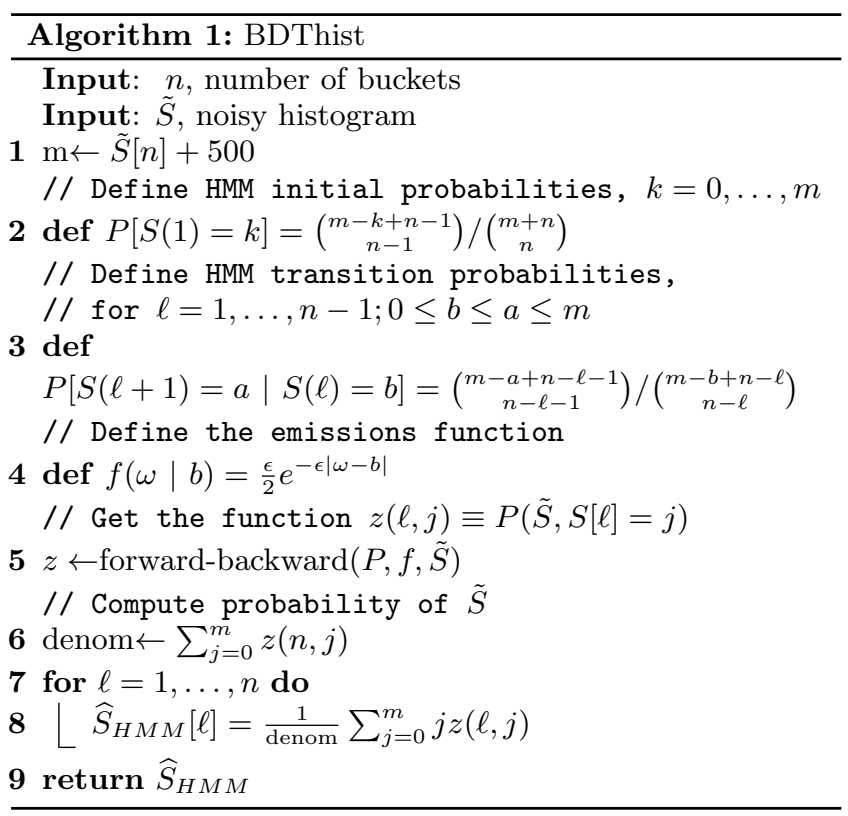

changing negative estimated cell counts to 0 . Note that the counts in this estimator are generally not integers. Also note that this method does not use knowledge of the noise distribution. Thus we also consider another baseline: a maximum likelihood estimator $\widehat{S}_{\mathrm{ML}}$ (using the pool-adjacent violators algorithm $[8,41,42])$ which is essentially the same as orderconstrained $L_{1}$ regression [8, 41, 42]. Our competing estimation algorithm is described in Section 7.2.

\subsection{The Algorithm}

Applying Bayesian decision theory to the sorted histogram problem requires choosing a prior distribution, a set of actions, an error function $\mathcal{E}$, and finally an algorithm to select the best action given the noisy histogram $\tilde{S}$. We note that the Bayesian approximation algorithm proposed by Williams and McSherry [46] is not applicable. First, [46] requires that the likelihood of the true input be modeled as an independent product distribution; this is not true of sorted histograms (in fact, we ended up modeling it as a Markov chain). Second, [46] provides an approximation scheme while our algorithm is exact. The estimation procedure is shown in Algorithm 1. In the rest of the section, we explain the algorithm and its derivation.

\subsubsection{Choice of prior (Lines 1, 2, 3)}

To ensure that our algorithm does not receive an unfair advantage (e.g., we should not use our knowledge of the distribution of degree sequences in typical social networks), we chose a uniform prior over sorted histograms with $n$ cells. In order to do this, we first need an upper bound $m$ on the maximum value of any cell in the true sorted histogram $S$. We set $m=500+\max _{j} \tilde{S}(j)$ (where $\tilde{S}$ is the publicly released noisy histogram). The addition of 500 ensures that for all choices of $\epsilon$ (parameter of the noise, see Section 7.1) between 0.1 and $1, m$ is larger than the true maximum of $S$ with probability $\approx 1$ (up to 22 decimal places).

There are $\left(\begin{array}{c}m+n \\ n\end{array}\right)$ sorted histograms with $n$ cells and at most $m$ elements per cell. Under our prior each one is 
equally likely. The crucial point is to recognize that this prior can be expressed as a non-stationary Markov chain.

A non-stationary Markov chain has a notion of time $\ell$, a state $S(\ell)$ at time $\ell$, an initial distribution $P(S(1)=k$ ) at time $\ell=1$, a transition probability $P(S(\ell+1)=a \mid S(\ell)=$ $b)$, and a requirement called the Markov property: $P(S(\ell+$ 1) $\mid S(\ell))=P(S(\ell+1) \mid S(\ell), S(\ell-1), \ldots, S(1))$.

To see how the uniform prior over sequences can be modeled as a Markov chain, we let time correspond to bucket id (which ranges from 1 to $n$ ) and we let the state at time $\ell$ be the count in bucket $\ell$, which, by abuse of notation, we also refer to as $S(\ell)$. The initial probability is given by Line 2 of the algorithm (the numerator is the number of nondecreasing sequences that have count $k$ in the first bucket and the denominator is the total number of nondecreasing sequences). The transition probabilities are given by Line 3 . This is because each partial histogram for which we know $S(1), \ldots, S(\ell)$ and for which $S(\ell)=b$ can be extended to a nondecreasing sequence with $n$ buckets in $\left(\begin{array}{c}m-b+n-\ell \\ n-\ell\end{array}\right)$ ways. For $\left(\begin{array}{c}m-a+n-\ell-1 \\ n-\ell-1\end{array}\right)$ of those ways, bucket $\ell+1$ has count $S(\ell+1)=a$. Note that both quantities are independent of the values of $S(1), \ldots, S(\ell-1)$ which implies that the Markov property holds and that the transition probability is their ratio (i.e. is shown in Line 3 ).

Note that we never see the true sorted histogram and thus we never get to observe the states. Instead, we observe noisy values $\tilde{S}(1), \ldots, \tilde{S}(n)$ where the conditional distribution of the noisy count $P(\tilde{S}(i) \mid S(1), \ldots, S(n))$ is equal to $P(\tilde{S}(i) \mid S(i))$ and has the density $f(x)=\frac{e}{2} e^{-|x-S(i)|}$ since Laplace noise is added to each bucket. This is referred to as an emission probability and the overall process with the Markov chain prior and the emission probabilities is called a hidden Markov model [9].

\subsubsection{Choice of the set $\mathbb{A}$ of actions}

The constrained least-squares regression in [24] produced a sorted histogram whose cell entries do not have to be integers. Likewise we set $\mathbb{A}$, the set of actions, to be the set of all sorted histograms whose cell entries are bounded by $m$ but are not necessarily integers (so an action $a_{\widehat{S}} \in \mathbb{A}$ corresponds to using $\widehat{S}$ as the estimator of the true sorted histogram).

\subsubsection{Choice of error function}

The constrained least-squares regression in [24] seeks to minimize the sum squared error between the noisy output $\tilde{S}$ and the candidate estimator $\widehat{S}$. We use this sum squared error as our choice of error function $\mathcal{E}$. This is also the error measure we will use to experimentally evaluate the quality of an estimator (out of fairness to the constrained least-squares regression, since that is what it attempts to optimize).

\subsubsection{Computing the Estimator (Lines 4-8)}

To use Bayesian decision theory, we need to take the noisy output $\tilde{S}$ and produce an action (sorted histogram) with least cost, as specified in Equation 3 (Section 6.1). Since the error function $\mathcal{E}$ is just sum-squared error, it is wellknown [9] that the optimal action (sorted histogram) is the expectation of the posterior distribution:

$$
E[S \mid \tilde{S}]=\sum_{S \in \mathbb{I}} S \times P(\text { data }=S \mid \mathfrak{M}(\text { data })=\tilde{S})
$$

Note that here $\mathbb{I}$ is the set of sorted histograms, and $S \in \mathbb{I}$ is treated mathematically as a vector of dimension $n$. Equation 4 is indeed a sorted histogram as it is the weighted average of sorted histograms.

Lines 4-8 of Algorithm 1 are designed to compute this value as follows. Since $S$ is modeled as a non-stationary Markov chain, then the noisy version $\tilde{S}$ becomes a hidden Markov model (HMM) with the same state transitions as $S$ and with emissions probabilities specified by the Laplace $(1 / \epsilon)$ distribution. The forward-backward [9, 37] algorithm for HMMs will produce the value of $P(\tilde{S}, S[\ell]=j)$ for all $\ell$ and $j$ simultaneously; it is stored as the function $z(\ell, j)$. This is done in Line 5 . Then Line 6 computes $P(\tilde{S})$. Now note that $z(\ell, j) / P(\tilde{S})=P(S[\ell]=j \mid \tilde{S})$. Using this fact, the loop on Lines 7 and 8 computes the expected value $E[S[\ell] \mid \tilde{S}]$ so that the return value $\widehat{S}_{H M M}$ is the desired expectation from Equation 4.

\section{EXPERIMENTS}

We now compare our estimator (based on Bayesian decision theory) for the sorted histogram problem against the baselines of least-squares estimation [24] and maximum likelihood estimation. These experiments can also be viewed as a partial validation of the axiomatic approach to utility, since it implied that we should be using Bayesian decision theory to work with sanitized data.

\subsection{The Setup}

\subsubsection{Datasets}

For comparison with prior work [24], we evaluate the estimators for the sorted histogram using the three datasets considered by Hay et al. [24]. These are the social network dataset (consisting of 11,342 histogram cells), the net trace dataset (consisting of 65, 536 histogram cells), and the search logs dataset (consisting of 32,768 histogram cells).

\subsubsection{The Baselines}

Let $\widehat{S}_{H M M}$ denote the estimator for the sorted histogram problem that is based on Bayesian decision theory (as described in Section 7.2). We compare our estimator against a maximum likelihood estimator $\widehat{S}_{M L}$. Because Laplace noise was added to the original sorted histogram $S$ to get the noisy histogram $\tilde{S}$, a maximum likelihood estimator is simply the sorted histogram that is closest to $\tilde{S}$ in the $L_{1}$ distance. It can be found using the pool-adjacent violators (PAV) algorithm $[8,41,42]$. We also compare against $\widehat{S}_{L S}$, the original solution of Hay et al. which returned the sorted histogram that is closest to $\tilde{S}$ in terms of sum-squared error (i.e. $L_{2}$ distance).

\subsubsection{Implementation}

We ran all of our experiments on computing clusters that used a variety configurations of the compute nodes, ranging from 4-6 core, 2.66-3.06 GhZ, and 48-96GB RAM. The code for $\widehat{S}_{L S}$ (from [24]) was written in Python, as was our implementation of the $\widehat{S}_{M L}$, the maximum likelihood estimator. Our new estimator $\widehat{S}_{H M M}$, based on Bayesian decision theory, was written in $\mathrm{C}$ - this is because it is significantly slower (the apparent cost for improved accuracy) and we needed to run thousands of experimental runs for this paper. For this reason we will later analyze both time complexity and wall-clock running time. 

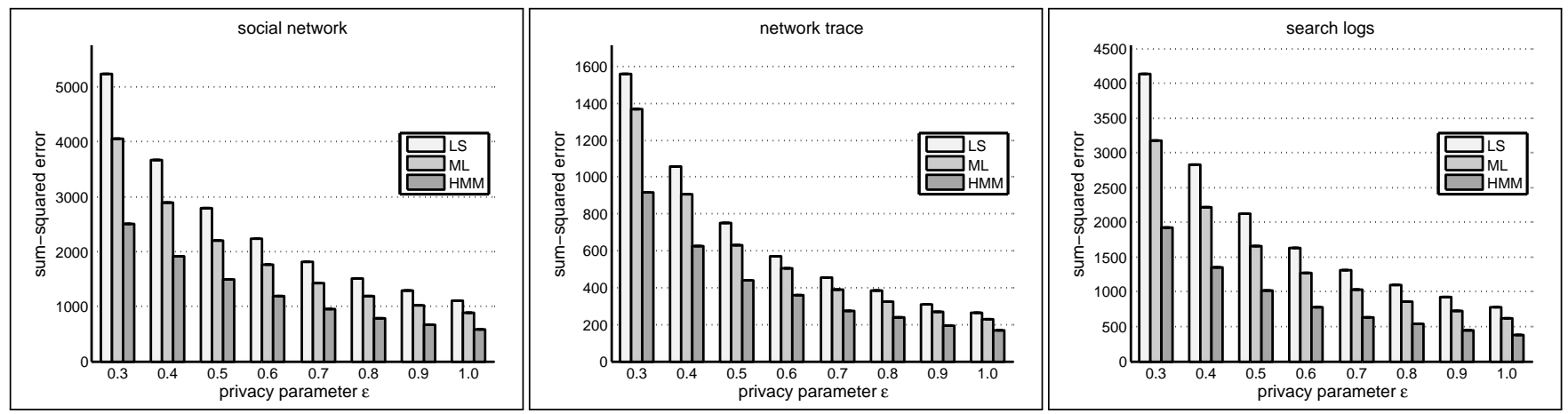

Figure 1: Sum-squared error of the estimators $\widehat{S}_{L S}, \widehat{S}_{M L}, \widehat{S}_{H M M}$ averaged over 100 runs for each $\epsilon$. Average error of $\widehat{S}_{H M M}$ outperformed baselines by at least $26 \%$.

\begin{tabular}{|cl||c|c|c|c|c|c|c|c|c|c|}
\hline & Estimator & 1.0 & 0.9 & 0.8 & 0.7 & 0.6 & 0.5 & 0.4 & 0.3 & 0.2 & 0.1 \\
\hline \multirow{3}{*}{ social network } & $\widehat{S}_{\mathrm{HMM}}$ & $\mathbf{1 0 0}$ & $\mathbf{1 0 0}$ & $\mathbf{1 0 0}$ & $\mathbf{1 0 0}$ & $\mathbf{1 0 0}$ & $\mathbf{1 0 0}$ & $\mathbf{1 0 0}$ & $\mathbf{1 0 0}$ & $\mathbf{1 0 0}$ & $\mathbf{1 0 0}$ \\
& $\widehat{S}_{\mathrm{LS}}$ & 0 & 0 & 0 & 0 & 0 & 0 & 0 & 0 & 0 & 0 \\
& $\widehat{S}_{\mathrm{ML}}$ & 0 & 0 & 0 & 0 & 0 & 0 & 0 & 0 & 0 & 0 \\
\hline \hline \multirow{3}{*}{ network trace } & $\widehat{S}_{\mathrm{HMM}}$ & $\mathbf{1 0 0}$ & $\mathbf{1 0 0}$ & $\mathbf{1 0 0}$ & $\mathbf{1 0 0}$ & $\mathbf{1 0 0}$ & $\mathbf{1 0 0}$ & $\mathbf{1 0 0}$ & $\mathbf{1 0 0}$ & $\mathbf{1 0 0}$ & $\mathbf{9 9}$ \\
& $\widehat{S}_{\mathrm{LS}}$ & 0 & 0 & 0 & 0 & 0 & 0 & 0 & 0 & 0 & 0 \\
& $\widehat{S}_{\mathrm{ML}}$ & 0 & 0 & 0 & 0 & 0 & 0 & 0 & 0 & 0 & 1 \\
\hline \hline \multirow{3}{*}{ search logs } & $\widehat{S}_{\mathrm{HMM}}$ & $\mathbf{1 0 0}$ & $\mathbf{1 0 0}$ & $\mathbf{1 0 0}$ & $\mathbf{1 0 0}$ & $\mathbf{1 0 0}$ & $\mathbf{1 0 0}$ & $\mathbf{1 0 0}$ & $\mathbf{1 0 0}$ & $\mathbf{1 0 0}$ & $\mathbf{1 0 0}$ \\
& $\widehat{S}_{\mathrm{LS}}$ & 0 & 0 & 0 & 0 & 0 & 0 & 0 & 0 & 0 & 0 \\
& $\widehat{S}_{\mathrm{ML}}$ & 0 & 0 & 0 & 0 & 0 & 0 & 0 & 0 & 0 & 0 \\
\hline
\end{tabular}

Table 1: For each dataset and $\epsilon$ value, we show the number of times (out of 100) a particular estimator had the lowest sumsquared error.

\subsection{Results}

We now compare the three estimators $\widehat{S}_{H M M}, \widehat{S}_{M L}$, and $\widehat{S}_{L S}$ on the three datasets social network, network trace, and search logs considered by [24].

\subsubsection{Accuracy}

The baseline estimator $\widehat{S}_{L S}$ (from [24]) was designed to minimize sum-squared error and thus our experiments compare the sum-squared error between an estimator and the true sorted histogram $S$.

Our first results are shown in Figure 1. For each combination of dataset and $\epsilon$ value, we generated 100 noisy histograms $\tilde{S}_{1}, \ldots, \tilde{S}_{100}$ by adding Laplace $(1 / \epsilon)$ noise to each. For each of the hundred $\tilde{S}_{i}$, we computed the estimators $\widehat{S}_{L S}, \widehat{S}_{M L}, \widehat{S}_{H M M}$ and then computed the sum-squared error between the estimators and the true original sorted histogram. The values shown in Figure 1 are averaged over those 100 repetitions. It is clear that $\tilde{S}_{H M M}$ outperforms the other estimators. For each dataset and $\epsilon$ value, the average error of $\widehat{S}_{H M M}$ was at least $26 \%$ less than the error of the others. We consider statistical significance next.

\subsubsection{Statistical Significance and Consistency}

For each dataset and $\epsilon$ value, we generated 100 noisy histograms $\tilde{S}_{1}, \ldots, \tilde{S}_{100}$. For each $\tilde{S}_{i}$, we determined which of the 3 estimators had lowest error. We tabulated the results in Table 1. As we can see, $\widehat{S}_{H M M}$ was consistently the best estimator, outperforming the others 100 out of 100 times for almost all experimental settings (the only exception was the network trace dataset with $\epsilon=0.1$ for which it was the best 99 out of 100 times). This is highly statistically significant with $p$-value less than $10^{-29}$.

\subsubsection{Time Complexity and Running times}

The time complexity of constrained least-squares regression for producing $\widehat{S}_{\mathrm{LS}}$ is $O(n)$ [24], where $n$ is the number of cells in the sorted histogram. Maximum likelihood inference for producing $\widehat{S}_{\mathrm{ML}}$ used our $O(n \log n)$ implementation of the pool-adjacent violators (PAV) algorithm [8, 41, 42]. The forward-backward algorithm for producing $\widehat{S}_{\mathrm{HMM}}$ is $O\left(\mathrm{~nm}^{2}\right)$ where $m$ is the upper bound on the maximum value of any cell. Thus we see that improvements in accuracy come at the cost of running time. Experimentally, we saw that the running time for computing $\widehat{S}_{\mathrm{HMM}}$ was $\approx 20$ hours for network trace, $\approx 2.5$ hours for search logs, and $\approx 4.5$ hours for social network. On the other hand, computing $\widehat{S}_{\mathrm{LS}}$ and $\widehat{S}_{\mathrm{ML}}$ often took less than a second (except for $\widehat{S}_{\mathrm{ML}}$ on network trace, which took $<5$ seconds on average) .

\subsubsection{Intangibles}

Our experimental results showed that the improved estimation accuracy came at the cost of a higher running time. In general, for sophisticated end-users there are other considerations. Extracting utility from sanitized data is nontrivial: the constrained least-squares regression estimator $\widehat{S}_{L S}$ relies on a clever algorithm [24] as does the PAV algorithm [8, 41, $42]$ for the maximum likelihood estimator $\widehat{S}_{M L}$. Our HMMbased estimator relies on the nontrivial forward-backward dynamic programming algorithm with scaling factors for numerical stability [9]. Overall this points to exciting research opportunities in the statistical analysis of sanitized data.

\section{CONCLUSIONS}

In this paper we presented a new estimation algorithm for the sorted histogram that outperforms previous approaches. 
The insight behind this estimation procedure came from a new analysis of utility axioms showing that the amount of information preserved by a sanitizing algorithm should be measured as the expected error of a Bayesian decision maker (hence Bayesian decision theory should play an important role in processing sanitized data). Key areas of future work include efficient Bayesian algorithms for the complex noise distributions created by data sanitizers, improved strategies for designing sanitizing algorithms for maximizing information retention, and efficient algorithms for estimating the amount of information retained.

\section{ACKNOWLEDGMENTS}

This material is based upon work supported by the National Science Foundation under Grant No. 1054389.

\section{REFERENCES}

[1] John M. Abowd and Simon D. Woodcock. Disclosure limitation in longitudinal linked data. Confidentiality, Disclosure, and Data Access: Theory and Practical Applications for Statistical Agencies, pages 215-277, 2001.

[2] Nabil Adam and John Worthmann. Security-control methods for statistical databases. ACM Computing Surveys, 21(4):515-556, 1989.

[3] Charu C. Aggarwal. On unifying privacy and uncertain data models. In $I C D E, 2008$.

[4] Mário S. Alvim, Miguel E. Andrés, Konstantinos Chatzikokolakis, Pierpaolo Degano, and Catuscia Palamidessi. Differential privacy: on the trade-off between utility and information leakage. http://arxiv.org/abs/1103.5188, 2011.

[5] Mário S. Alvim, Miguel E. Andrés, Konstantinos Chatzikokolakis, and Catuscia Palamidessi. On the relation between differential privacy and quantitative information flow. In $I C A L P, 2011$.

6] Mina Askari, Reihaneh Safavi-Naini, and Ken Barker. An information theoretic privacy and utility measure for data sanitization mechanisms. In CODASPY, 2012.

[7] Boaz Barak, Kamalika Chaudhuri, Cynthia Dwork, Satyen Kale, Frank McSherry, and Kunal Talwar. Privacy, accuracy and consistency too: A holistic solution to contingency table release. In PODS, 2007.

[8] R. E. Barlow, D. J. Bartholomew, J. M. Bremner, and H. D. Brunk. Statistical Inference Under Order Restrictions. John Wiley and Sons, 1972.

[9] Christopher M. Bishop. Pattern Recognition and Machine Learning. Springer, 2006.

[10] Avrim Blum, Cynthia Dwork, Frank McSherry, and Kobbi Nissim. Practical privacy: the SuLQ framework. In PODS, pages 128-138, 2005.

[11] Avrim Blum, Katrina Ligett, and Aaron Roth. A learning theory approach to non-interactive database privacy. In STOC, pages 609-618, 2008.

[12] Stephen Boyd and Lieven Vandenberghe. Convex Optimization. Cambridge University Press, New York, NY, USA, 2004.

[13] Bee-Chung Chen, Daniel Kifer, Kristen LeFevre, and Ashwin Machanavajjhala. Privacy-preserving data publishing. Foundations and Trends in Databases, 2(1-2):1-167, 2009.

[14] Bolin Ding, Marianne Winslett, Jiawei Han, and Zhenhui Li. Differentially private data cubes: optimizing noise sources and consistency. In SIGMOD, 2011.

[15] Irit Dinur and Kobbi Nissim. Revealing information while preserving privacy. In PODS, 2003.

[16] Cynthia Dwork, Frank McSherry, Kobbi Nissim, and Adam Smith. Calibrating noise to sensitivity in private data analysis. In $T C C, 2006$.
[17] Cynthia Dwork, Moni Naor, Omer Reingold, Guy N.Rothblum, and Salil Vadhan. On the complexity of differentially private data release: Efficient algorithms and hardness results. In $S T O C, 2009$.

[18] Alexandre Evfimievski, Ramakrishnan Srikant, Rakesh Agrawal, and Johannes Gehrke. Privacy preserving mining of association rules. In KDD, 2002.

[19] B. C. M. Fung, K. Wang, R. Chen, and P. S. Yu. Privacy-preserving data publishing: A survey on recent developments. ACM Computing Surveys, 42(4), 2010.

[20] A. Gelman, J. B. Carlin, H. S. Stern, and D. B. Rubin. Bayesian Data Analysis. Chapman \& Hall/CRC, 2nd edition, 2003.

[21] Arpita Ghosh and Aaron Roth. Selling privacy at auction. In $E C, 2011$.

[22] Arpita Ghosh, Tim Roughgarden, and Mukund Sundararajan. Universally utility-maximizing privacy mechanisms. In STOC, 2009.

[23] Mangesh Gupte and Mukund Sundararajan. Universally optimal privacy mechanisms for minimax agents. In PODS, 2010.

[24] M. Hay, V. Rastogi, G. Miklau, and D. Suciu. Boosting the accuracy of differentially-private histograms through consistency. In $V L D B, 2010$.

[25] Ali Inan, Murat Kantarcioglu, and Elisa Bertino. Using anonymized data for classification. In ICDE, 2009

[26] Vijay S. Iyengar. Transforming data to satisfy privacy constraints. In $K D D, 2002$.

[27] Daniel Kifer and Bing-Rong Lin. Towards an axiomatization of statistical privacy and utility. In $P O D S$, 2010.

[28] Daniel Kifer and Bing-Rong Lin. An axiomatic view of statistical privacy and utility. Journal of Privacy and Confidentiality, 4(1), 2012.

[29] Chao Li, Michael Hay, Vibhor Rastogi, Gerome Miklau, and Andrew McGregor. Optimizing linear counting queries under differential privacy. In PODS, 2010.

[30] Chao Li, Daniel Yang Li, Gerome Miklau, and Dan Suciu. A theory of pricing private data. In ICDT, 2013.

[31] Roderick J.A. Little. Statistical analysis of masked data. Journal of Official Statistics, 9(2):407-426, 1993.

[32] Frank McSherry and Kunal Talwar. Mechanism design via differential privacy. In FOCS, 2007.

[33] Frank D. McSherry. Privacy integrated queries: An extensible platform for privacy-preserving data analysis. In SIGMOD, pages 19-30, 2009.

[34] Roger B. Myerson. Axiomatic foundations of bayesian decision theory. Technical Report 671, Northwestern University Center for Mathematical Studies in Economics and Management Science, 1986.

[35] J. B. Paris. The Uncertain Reasoner's Companion. Cambridge University Press, 1994.

[36] Davide Proserpio, Sharon Goldberg, and Frank McSherry. A workflow for differentially-private graph synthesis. In SIGCOMM Workshop on Online Social Networks, 2012.

[37] L. Rabiner. A tutorial on hidden markov models and selected applications in speech recognition. Proceedings of the IEEE, 77(2):257-286, 1989.

[38] T.E. Raghunathan, J.P. Reiter, and D.B. Rubin. Multiple imputation for statistical disclosure limitation. Journal of Official Statistics, 19:1-16, 2003.

[39] Vibhor Rastogi, Dan Suciu, and Sungho Hong. The boundary between privacy and utility in data publishing. In $V L D B, 2007$.

[40] J.P. Reiter. Using cart to generate partially synthetic public use microdata. Journal of Official Statistics, pages 441-462, 2005.

[41] Tim Robertson and Paul Waltman. On estimating monotone parameters. Ann. Math. Statist., 39(3):1030-1039, 1968.

[42] Tim Robertson and F. T. Wright. Algorithms in order 
restricted statistical inference and the cauchy mean value property. Ann. Statist., 8(3):645-651, 1980.

[43] Leonard J. Savage. The Foundations of Statistics. Dover 1972.

[44] John von Neumann and Oskar Morgenstern. Theory of Games and Economic Behavior. Princeton University Press, 2007.

[45] Larry Wasserman. All of Statistics: A Concise Course in Statistical Inference. Springer, 2003.

[46] Oliver Williams and Frank McSherry. Probabilistic inference and differential privacy. In NIPS, 2010.

[47] Raymond Wong, Ada Fu, Ke Wang, and Jian Pei. Minimality attack in privacy preserving data publishing. In $V L D B, 2007$.

[48] Xiaokui Xiao and Yufei Tao. Anatomy: Simple and effective privacy preservation. In $V L D B, 2006$.

[49] Qing Zhang, Nick Koudas, Divesh Srivastava, and Ting Yu. Aggregate query answering on anonymized tables. In ICDE, 2007.

\section{APPENDIX}

\section{A. PROOF OF THEOREM 5.3}

THEOREM 5.3. A utility $\mu_{\mathbb{I}}{ }^{S E}$ or loss $\mathcal{L}_{\mathbb{I}}{ }^{S E}$ measure (as described in Definition 5.1) satisfies Axiom 4.1 (sufficiency) only if it is a constant function.

PROOF. We will show that $\mu_{\mathbb{I}}{ }^{\mathrm{SE}}$ satisfies Axiom 4.1 (sufficiency) if and only if:

$$
\begin{aligned}
Q\left(D, \omega_{1}\right)= & Q\left(D, \omega_{2}\right), \forall \omega_{1}, \omega_{2} \in \operatorname{range}(\mathfrak{M}), \\
& \forall D \in \mathbb{I} \text { with } P(D)>0
\end{aligned}
$$

It is clear that such a $Q$ implies a constant $\mu_{\mathbb{I}} \mathrm{SE}$

By way of contradiction, suppose that there exist $\omega_{1}, \omega_{2} \in$ range $(\mathfrak{M})$ and a $D \in \mathbb{I}$ such that $Q\left(D, \omega_{1}\right)<Q\left(D, \omega_{2}\right)$ and $P(D)>0$. Choose any $p$ such that $1>p>0$. Let $\mathfrak{M}_{1}$ and $\mathfrak{M}_{2}$ behave as follows.

$$
\begin{array}{r}
P\left(\mathfrak{M}_{1}(D)=\omega_{1}\right)=p \\
P\left(\mathfrak{M}_{1}(D)=\omega_{2}\right)=1-p \\
P\left(\mathfrak{M}_{1}\left(D^{\prime}\right)=\omega_{2}\right)=1, \forall D^{\prime} \in \mathbb{I}, D^{\prime} \neq D \\
P\left(\mathfrak{M}_{2}\left(D^{\prime}\right)=\omega_{2}\right)=1, \forall D^{\prime} \in \mathbb{I}
\end{array}
$$

Let $\mathcal{A}$ be an algorithm that always outputs $\omega_{2}$. Then $\mathfrak{M}_{2}=$ $\mathcal{A} \circ \mathfrak{M}_{1}$. Using the assumption of $Q\left(D, \omega_{1}\right)<Q\left(D, \omega_{2}\right)$ :

$$
\begin{aligned}
& \mu_{\mathbb{I}}^{\mathrm{SE}}\left(\mathfrak{M}_{1}\right)-\mu_{\mathbb{I}}^{\mathrm{SE}}\left(\mathfrak{M}_{2}\right) \\
= & P(D) p Q\left(D, \omega_{1}\right)+P(D)(1-p) Q\left(D, \omega_{2}\right)-P(D) Q\left(D, \omega_{2}\right) \\
= & P(D) p\left(Q\left(D, \omega_{1}\right)-Q\left(D, \omega_{2}\right)\right)<0
\end{aligned}
$$

since $P(D)>0$ and $p>0$. This contradicts the axiom of sufficiency.

\section{B. PROOF OF THEOREM 5.4}

THEOREM 5.4. Let $\mathbb{I}$ be finite. A utility $\mu_{\mathbb{I}}{ }^{S M}$ or loss $\mathcal{L}_{\mathbb{I}}{ }^{S M}$ measure (as described in Definition 5.2) satisfies Axiom 4.1 (sufficiency) only if it is a constant function.

Proof. Suppose $\mu_{\mathbb{I}}{ }^{\mathrm{SM}}$ satisfies Axiom 4.1. Choose an ordering $\omega_{1}, \omega_{2}, \ldots$ for the elements of $\mathbb{O}^{*}$.

Part 1: Showing $\min _{D \in \mathbb{I}} Q(D, \omega)$ is a constant $\gamma$ independent of $\omega$. For each $\omega$, define the data sanitizer $\mathfrak{M}_{\omega}$ that always outputs $\omega$. Clearly, for any data sanitizer $\mathfrak{M}$, there exists a $\mathcal{A}$ such that $\mathfrak{M}_{\omega}=\mathcal{A} \circ \mathfrak{M}$ and so by Axiom 4.1 of sufficiency, $\mu_{\mathbb{I}}^{\mathrm{SM}}\left(\mathfrak{M}_{\omega}\right)$ is the same for all $\omega$ and corresponds to the minimum value $\mu_{\mathbb{I}}{ }^{\mathrm{SM}}$ can take. Call this value $\gamma$.

By definition, $\mu_{\mathbb{I}}{ }^{\mathrm{SM}}\left(\mathfrak{M}_{\omega}\right)=\gamma=\min _{D \in \mathbb{I}} Q(D, \omega)$ no matter which $\omega$ we choose.

Part 2: Showing that for every $k$, there exists a $D \in \mathbb{I}$ such that $Q\left(D, \omega_{1}\right)=Q\left(D, \omega_{2}\right)=\cdots=Q\left(D, \omega_{k}\right)=\gamma$

For each $k=1,2, \ldots$ define the data sanitizer $\mathfrak{M}_{k}$ as follows:

$$
P\left(\mathfrak{M}_{k}(D)=\omega_{i}\right)= \begin{cases}1 / k & \text { if } i \leq k \\ 0 & \text { otherwise }\end{cases}
$$

Thus $\mathfrak{M}_{k}$ ignores its input and returns one of $\omega_{1}, \ldots, \omega_{k}$ uniformly at random. Since the $\mathfrak{M}_{k}(k=1,2, \ldots)$ ignore their inputs then clearly for any data sanitizer $\mathfrak{M}$, there exists an algorithm $\mathcal{A}$ with $\mathfrak{M}_{k}=\mathcal{A} \circ \mathfrak{M}$ and so, if the axiom of sufficiency is to hold, $\mu_{\mathbb{I}}{ }^{\mathrm{SM}}\left(\mathfrak{M}_{k}\right)=\gamma$ (the minimum value of $\left.\mu_{\mathbb{I}} \mathrm{SM}\right)$ for all $k$. Since $\gamma$ is the minimum value $Q(D, \omega)$ can take (for any $D$ and $\omega$ ), then by definition of $\mu_{\mathbb{I}}$ SM , the dataset $D$ that achieves the minimum in Equation 1 (in Definition 5.2) must have $Q\left(D, \omega_{1}\right)=Q\left(D, \omega_{2}\right)=\cdots=$ $Q\left(D, \omega_{k}\right)=\gamma$

Part 3: Showing that there exists a $D^{*} \in \mathbb{I}$ such that $Q\left(D^{*}, \omega\right)=\gamma$ for all $\omega$. If $\left\{\omega_{1}, \omega_{2}, \ldots\right\}$ is finite, this follows from Part 2. If it is countably infinite, then suppose on the contrary that for every $D$ there was an $\omega_{i_{D}}$ such that $Q\left(D, \omega_{i_{D}}\right)>\gamma$. Then since $\mathbb{I}$ is finite, we have a contradiction with Part 2 by setting $k=1+\max \left\{i_{D}: D \in \mathbb{I}\right\}$.

This $D^{*}$ is the minimizer of Equation 1 in Definition 5.2. Hence $\mu_{\mathbb{I}}{ }^{\mathrm{SM}}(\mathfrak{M})=\gamma$ for all $\mathfrak{M}$.

\section{PROOF OF THEOREM 6.2}

To prove theorem 6.2 , we need the following technical result from [28]:

Lemma C.1 ([28]). A loss measure $\mathcal{L}_{\mathbb{I}}$ satisfies Axioms 4.1, 4.3, and 4.4 if and only if $\mathcal{L}_{\mathbb{I}}$ has the form:

$\mathcal{L}_{\mathbb{I}}(\mathfrak{M})=\sum_{\omega \in \operatorname{range}(\mathfrak{M})} f\left(P\left(\mathfrak{M}\left(D_{1}\right)=\omega, \ldots, P\left(\mathfrak{M}\left(D_{n}\right)=\omega\right)\right)\right.$

where $f$ is a continuous function that is concave over vectors with no negative components and $f(c \vec{x})=c f(\vec{x})$ for all nonnegative $c$.

THEOREM 6.2. Let $\mathbb{I}$ be finite.

(i) For every choice of $\mathbb{A}$, prior over $\mathbb{I}$, and error function $\mathcal{E}$ that is bounded from below, the resulting loss measure $\mathcal{L}_{\mathbb{I}}{ }^{B D T}$ (Definition 6.1) satisfies Axioms 4.1, 4.3, and 4.4 .

(ii) If a loss measure $\mathcal{L}_{\mathbb{I}}$ satisfies Axioms 4.1, 4.3, and 4.4 then there exists a prior over $\mathbb{I}$, a set of actions $\mathbb{A}$, and an error function $\mathcal{E}$ (that is bounded from below) such the corresponding loss measure $\mathcal{L}_{\mathbb{I}}{ }^{B D T}$ equals $\mathcal{L}_{\mathbb{I}}$.

\section{Proof of $(i)$ :}

We must first show that $\mathcal{L}_{\mathbb{I}}{ }^{\mathrm{BDT}}$ is always finite and then we must show that Lemma C.1 applies. Let $a^{\prime}$ be any action.

$\mathcal{L}_{\mathbb{I}}{ }^{\mathrm{BDT}}(\mathfrak{M}) \leq \sum_{\omega \in \operatorname{range}(\mathfrak{M})} \sum_{D \in \mathbb{I}} P(D) P(\mathfrak{M}(D)=\omega) \mathcal{E}\left(D, a^{\prime}\right)<\infty$ 
because $\mathbb{I}$ is a finite set. It is also clear that $\mathcal{L}_{\mathbb{I}}{ }^{\mathrm{BDT}}(\mathfrak{M}) \geq$ $\beta>-\infty$, where $\beta$ is the lower bound on $\mathcal{E}$, so $\mathcal{L}_{\mathbb{I}}{ }^{\text {BDT }}$ is finite. To show that Lemma C.1 applies, notice that

$\mathcal{L}_{\mathbb{I}}^{\mathrm{BDT}}(\mathfrak{M})=\sum_{\omega \in \operatorname{range}(\mathfrak{M})} \inf _{a \in \mathbb{A}} \sum_{D \in \mathbb{I}} P(D) P(\mathfrak{M}(D)=\omega) \mathcal{E}(D, a)$

and so we must show that the function $f$ defined as

$$
\begin{aligned}
f & \left(P\left(\mathfrak{M}\left(D_{1}\right)=\omega, \ldots, P\left(\mathfrak{M}\left(D_{n}\right)=\omega\right)\right)\right. \\
\quad= & \inf _{a \in \mathbb{A}} \sum_{D \in \mathbb{I}} P(D) P(\mathfrak{M}(D)=\omega) \mathcal{E}(D, a)
\end{aligned}
$$

is continuous, concave over vectors with no negative components and $f(c \vec{x})=c f(\vec{x})$ for all nonnegative $c$. The function $f$ is concave because the infimum of linear functions is concave. The other two properties are obvious and therefore, by Lemma C.1, $\mathcal{L}_{\mathbb{I}}{ }^{\mathrm{BDT}}$ satisfies the three axioms.

\section{Proof of $(i i)$ :}

Let $\mathcal{L}_{\mathbb{I}}$ be a loss measure satisfying the three axioms. By Lemma C.1 (replacing the $f$ in the lemma with $-F$ ),

$$
\mathcal{L}_{\mathbb{I}}(\mathfrak{M})=\sum_{\omega \in \operatorname{range}(\mathfrak{M})}-F(\vec{P}[\mathfrak{M}(\cdot)=\omega])
$$

where $F$ is continuous, convex over vectors with no negative components, and $c F(\vec{x})=F(c \vec{x})$ for $c \geq 0$.

Consider the epigraph of $F$, defined as the following subset of $\mathbb{R}^{|\mathbb{I}|} \times \mathbb{R}$ :

$\operatorname{epi}(F)=\{(\vec{x}, z): z \geq F(\vec{x}) \wedge \vec{x}[i] \geq 0, i=1, \ldots,|\mathbb{I}|\}$

This is a convex set. Note that for any $\overrightarrow{x^{*}}$ with no negative components, the vector $\left(\overrightarrow{x^{*}}, F\left(\overrightarrow{x^{*}}\right)\right)$ lies the boundary of the epigraph). By the supporting hyperplane theorem [12], for any given $\overrightarrow{x^{*}}$ there exists a supporting hyperplane defined by the triple $\left(\vec{a}_{x^{*}}, b_{x^{*}}, h_{x^{*}}\right)$, with the following properties:

$\langle 1\rangle\left(\vec{a}_{\overrightarrow{x^{*}}}, b_{\overrightarrow{x^{*}}}\right) \neq(\overrightarrow{0}, 0)$

$\langle 2\rangle \vec{a}_{\overrightarrow{x^{*}}} \cdot \overrightarrow{x^{*}}+b_{\overrightarrow{x^{*}}} F\left(\overrightarrow{x^{*}}\right)=h_{\overrightarrow{x^{*}}}$

$\langle 3\rangle \vec{a}_{x^{*}} \cdot \vec{x}+b_{x^{*}} z \geq h_{\overrightarrow{x^{*}}}$ for all other $(\vec{x}, z) \in \operatorname{epi}(F)$.

Now we need to prove several results about $\vec{a}_{x^{*}}, b_{\overrightarrow{x^{*}}}, h_{x^{*}}$.

Part ii-a: We must show that if $\overrightarrow{x^{*}}$ has only positive components then $b_{\overrightarrow{x *}}>0$.

For all $s \geq 0$, Property $\langle 3\rangle$ and the definition of epi $(F)$ implies $\vec{a}_{x^{*}} \cdot \vec{x}+b_{x^{*}}(s+F(x)) \geq h_{x^{*}}$ which is only true if $b_{\overrightarrow{x^{*}}} \geq 0$.

Now, if it were true that $b_{x^{*}}=0$ then Property $\langle 1\rangle$ would imply that $\vec{a}_{x^{*}} \neq \overrightarrow{0}$. Properties $\langle 3\rangle$ and $\langle 2\rangle$ would then imply $\vec{a}_{x^{*}} \cdot \vec{x} \geq h_{\overrightarrow{x^{*}}}=\vec{a}_{x^{*}} \cdot \overrightarrow{x^{*}}$ for all $\vec{x}$ with no negative components. For any $c \geq 0$, substituting $c \overrightarrow{x^{*}}$ for $\vec{x}$, we get $c \vec{a}_{x^{*}} \cdot \overrightarrow{x^{*}} \geq h_{\overrightarrow{x^{*}}}=\vec{a}_{\overrightarrow{x^{*}}} \cdot \overrightarrow{x^{*}}$ for all $c \geq 0$ and this can only be true if $\vec{a}_{x^{*}} \cdot \overrightarrow{x^{*}}=0$. This means that $\vec{a}_{\overrightarrow{x^{*}}}$ and $\overrightarrow{x^{*}}$ are mutually orthogonal and $h_{\overrightarrow{x^{*}}}=0$. However, when $\overrightarrow{x^{*}}$ has only positive components then for small enough $\gamma>0$, the vector $\vec{y}$ def $\overrightarrow{x^{*}}-\gamma \vec{a}_{\overrightarrow{x^{*}}}$ also has only positive components but then $\vec{a}_{x^{*}} \cdot \vec{y}=-\gamma\left\|\vec{a}_{x^{*}}\right\|_{2}^{2}<0$ (because $b_{x^{*}}=0$ implies $\vec{a}_{\overrightarrow{x *}} \neq \overrightarrow{0}$ by Property $\left.\langle 1\rangle\right)$. This is now a contradiction of Property $\langle 3\rangle$ (since we showed $b_{\overrightarrow{x^{*}}}=0$ implies $h_{x^{*}}=0$ ). Thus $b_{\overrightarrow{x^{*}}}>0$.

Part ii-b: We must show that if $\overrightarrow{x^{*}}$ has only positive components then $h_{\overrightarrow{x^{*}}}=0$.

Using Property $\langle 2\rangle$, then Property $\langle 3\rangle$ (along with properties of $F$ and the definition of epi $(F))$,

$$
\begin{aligned}
\vec{a}_{\overrightarrow{x^{*}}} \cdot \overrightarrow{x^{*}}+b_{\overrightarrow{x^{*}}} F\left(\overrightarrow{x^{*}}\right) & =h_{\overrightarrow{x^{*}}} \quad \text { and } \\
\vec{a}_{\overrightarrow{x^{*}}} \cdot c \overrightarrow{x^{*}}+b_{\overrightarrow{x^{*}}} F\left(c \overrightarrow{x^{*}}\right) & =c \times\left(\vec{a}_{\overrightarrow{x^{*}}} \cdot c \overrightarrow{x^{*}}+b_{\overrightarrow{x^{*}}} F\left(\overrightarrow{x^{*}}\right)\right) \geq h_{\overrightarrow{x^{*}}}
\end{aligned}
$$

for any $c \geq 0$. This implies that $h_{\overrightarrow{x^{*}}}=0$.

Part ii-c: We derive a representation of $F$ for vectors $\vec{x}$ with only positive components. For $\overrightarrow{x^{*}}$ with only positive components, $b_{\overrightarrow{x^{*}}}>0$ and $h_{\overrightarrow{x^{*}}}=0$ thus, without loss of generality, we may assume $b_{x^{*}}=1$ (by dividing $b_{x^{*}}$ and $\vec{a}_{\overrightarrow{x *}}$ by the appropriate positive constant). Then when $\overrightarrow{x^{*}}$ has only positive components, we have

$\left\langle 1^{*}\right\rangle \vec{a}_{x^{*}} \cdot \overrightarrow{x^{*}}+F\left(\overrightarrow{x^{*}}\right)=0$

$\left\langle 2^{*}\right\rangle \vec{a}_{\overrightarrow{x^{*}}} \cdot \vec{x}+F(\vec{x}) \geq 0$ for all $\vec{x}$ with no negative components. Define

$$
\begin{aligned}
\mathbb{A} & =\left\{\vec{a}_{\overrightarrow{x^{*}}}: \overrightarrow{x *} \text { has only positive components }\right\} \\
\mathcal{E}\left(D_{i},-\vec{a}_{x^{*}}\right) & =\overrightarrow{a_{x^{*}}}[i] \times|\mathbb{I}| \\
P\left(D_{i}\right) & =1 /|\mathbb{I}| \quad \text { for all } D_{i}
\end{aligned}
$$

Now let $\vec{x}$ and $\vec{y}$ be any two vectors with only positive components. Properties $\left\langle 1^{*}\right\rangle$ and $\left\langle 2^{*}\right\rangle$ imply that the corresponding $\vec{a}_{\vec{y}}, \vec{a}_{\vec{x}}, b_{\vec{y}}, b_{\vec{x}}$ satisfy:

$$
\begin{aligned}
-F(\vec{x}) & =\vec{a}_{\vec{x}} \cdot \vec{x} ; \quad-F(\vec{y})=\vec{a}_{\vec{y}} \cdot \vec{y} ; \quad-F(\vec{x}) \leq \vec{a}_{\vec{y}} \cdot \vec{x} \\
& \Rightarrow-F(\vec{x})=\inf _{\vec{a}_{\vec{y}} \in \mathbb{A}} \vec{a}_{\vec{y}} \cdot \vec{x}=\inf _{\vec{a} \vec{y} \in \mathbb{A}} \sum_{D_{i} \in \mathbb{I}} \vec{a}_{\vec{y}}[i] \vec{x}[i] \\
& \Rightarrow-F(\vec{x})=\inf _{a \in \mathbb{A}} \sum_{D_{i} \in \mathbb{I}} P\left(D_{i}\right) \vec{x}[i] \mathcal{E}\left(D_{i}, a\right)
\end{aligned}
$$

Part ii-d: Finishing the proof. Since $F$ is continuous, Equation 7 can be extended to all vectors with no negative components. Plugging this into Equation 6 will finish the proof once we show that $\mathcal{E}$ is bounded from below.

Suppose $\mathcal{E}$ is not bounded from below. Then there is a sequence of (not necessarily distinct) inputs: $D_{1}, D_{2}, D_{3}, \ldots$ and a sequence of actions $a_{1}, a_{2}, \ldots$ such that $\mathcal{E}\left(D_{i}, a_{i}\right) \leq$ $-3^{i}$. Define $\mathfrak{M}$ with range $(\mathfrak{M})=\left\{\omega_{*}, \omega_{1}, \omega_{2}, \omega_{3}, \ldots\right\}$ with the property that for $i=1,2, \ldots$,

$$
\begin{aligned}
P\left(\mathfrak{M}(D)=\omega_{i}\right) & = \begin{cases}3^{-i} & \text { if } D=D_{i} \\
0 & \text { otherwise }\end{cases} \\
P\left(\mathfrak{M}(D)=\omega_{*}\right) & =1-P\left(\mathfrak{M}(D) \in\left\{\omega_{1}, \omega_{2}, \ldots\right\}\right)
\end{aligned}
$$

It follows that

$$
\begin{gathered}
\mathcal{L}_{\mathbb{I}}(\mathfrak{M})=-F\left(\vec{P}\left[\mathfrak{M}(\cdot)=\omega_{*}\right]\right)+\sum_{i=1}^{\infty}-F\left(\vec{P}\left[\mathfrak{M}(\cdot)=\omega_{i}\right]\right) \\
=-F\left(\vec{P}\left[\mathfrak{M}(\cdot)=\omega_{*}\right]\right)+\sum_{i=1}^{\infty} \inf _{a \in \mathbb{A}} \sum_{D \in \mathbb{I}} P(D) P\left(\mathfrak{M}(D)=\omega_{i}\right) \mathcal{E}(D, a) \\
\leq-F\left(\vec{P}\left[\mathfrak{M}(\cdot)=\omega_{*}\right]\right)+\sum_{i=1}^{\infty} \sum_{D \in \mathbb{I}} P(D) P\left(\mathfrak{M}(D)=\omega_{i}\right) \mathcal{E}\left(D, a_{i}\right) \\
\leq-F\left(\vec{P}\left[\mathfrak{M}(\cdot)=\omega_{*}\right]\right)+\sum_{i=1}^{\infty}(1 /|I|)\left(3^{-i}\right)\left(-3^{i}\right)=-\infty
\end{gathered}
$$

This is a contradiction, so $\mathcal{E}$ is bounded from below. 